\title{
Adaptación ¿permanente? al cambio: Percepciones sobre la modalidad virtual de aprendizaje en la educación superior
}

\section{Permanent? Change adaptation: perceptions on e-learning experiences in higher education}

Presentación: 02/02/2021

Aprobación: 16/03/2021

\author{
María Marta Coria \\ Universidad Tecnológica Nacional - Argentina \\ mariamarta.coria@gmail.com
}

\section{Resumen}

El trabajo examina las valoraciones de estudiantes y docentes sobre la modalidad virtual de enseñanza-aprendizaje, las principales ventajas e inconvenientes percibidos, las preferencias sobre los formatos de clases y las herramientas didácticas que resultan más atractivas para aprender. Los resultados muestran que, tanto docentes como estudiantes, se adaptaron a la modalidad virtual a pesar de que la mayoría no tenía experiencias previas en esta modalidad de enseñanza-aprendizaje. Las principales ventajas percibidas respecto de la modalidad virtual son la flexibilidad del cursado mientras que las desventajas son los problemas de conectividad. Las prácticas docentes, en su mayoría, se adaptaron a la modalidad virtual, mejorando el desempeño de los estudiantes.

Palabras clave: educación superior, modalidad virtual, adaptación al cambio

\begin{abstract}
This paper examines perceptions on virtual learning, the advantages and disadvantages perceived by students and professors, as well as their preferences about traditional or virtual learning and the teaching tools used to improve academic performance. Results show that both students and professors adjusted to virtual learning despite the fact that most of them had no experience in that way of teaching. The main advantages perceived are flexibility and the main disadvantage is connectivity difficulties. Most teacher's practices were adjusted to virtual learning resulting in a better academic performance.
\end{abstract}

Key words: higher education, e-learning, change adaptation 


\section{INTRODUCCIÓN}

Las medidas dispuestas en 2020 en respuesta a la pandemia de COVID-19 generaron una serie de cambios en múltiples actividades, entre ellas la educación. En Argentina las clases presenciales se suspendieron el 16 de marzo de 2020 y las instituciones educativas, de todos niveles, debieron adaptarse para desarrollar una modalidad de enseñanza virtualizada.

En la educación superior, sin embargo, la modalidad virtual no era algo nuevo. Ésta viene atravesando en los últimos 25 años un proceso de diversificación, tanto de las carreras ofrecidas como de las modalidades a través de las cuales los estudiantes acceden a ella. La introducción de las tecnologías de la información y la comunicación permitieron generar ofertas de educación a distancia, modalidad que tuvo un crecimiento considerable en los últimos 20 años, tanto de la cantidad de carreras ofrecidas como de la cantidad de estudiantes. Diversas instituciones exploraron el modo de educación a distancia desde hace años y lograron consolidar sistemas de enseñanza que incluso cuentan con reconocimiento de la autoridad educativa nacional. Sobre este particular, en el año 2017, el Ministerio de Educación y Deportes de la Nación, mediante la Resolución $\mathrm{N}^{\circ} 2641$ aprueba el documento sobre la opción pedagógica y didáctica de educación a distancia. En dicho documento se definen aspectos sobre la estructura y cargas horarias de las carreras ofrecidas en la modalidad de educación a distancia, el procedimiento de evaluación y acreditación y posterior reconocimiento oficial y validez nacional de los títulos, entre otros aspectos relevantes. Además, las instituciones universitarias que incorporen o decidan incorporar la opción pedagógica y didáctica de educación a distancia, de acuerdo a la mencionada Resolución Ministerial $\mathrm{N}^{\circ} 2641 / 17$, deben presentar ante la Comisión Nacional de Evaluación y Acreditación Universitaria (CONEAU) los Sistemas Institucionales de Educación a Distancia (SIED) para que sean evaluados.

En el año 2017 había un total de 494 ofertas de carreras universitarias en modalidad a distancia, lo que representaba el 4\% del total (Ministerio de Educación de la Nación, 2017). Asimismo, en el año 2016 había en el sistema universitario 154.908 estudiantes de pregrado y grado en modalidad virtual, lo que representaba el 7,4\% del total de estudiantes universitarios (Ministerio de Educación de la Nación, 2017). De los estudiantes que cursan con modalidad a distancia, el 66\% corresponde al sector de gestión privada y el $34 \%$ al sector público.

En 2020 todas las instituciones universitarias debieron adaptar sus sistemas para poder desarrollar la totalidad de sus ofertas académicas en modalidad virtual, implementándose diferentes propuestas como las clases presenciales virtualizadas o modalidad virtual sincrónica o las propuestas que combinan clases en vivo a través de plataformas virtuales con actividades asincrónicas en plataformas de educación a distancia.

La adaptación a la virtualidad no fue sencilla ni estuvo exenta de dificultades, tanto de índole tecnológica como institucional y personal. La necesidad de continuar con el proceso pedagógico sin instancias presenciales generó demandas de mayor soporte institucional y tecnológico, puesta a punto de plataformas de educación a distancia, necesidad de apoyo docente, el desarrollo de una mayor capacidad de los estudiantes para realizar un aprendizaje guiado más autónomo, entre otros desafíos.

Habiendo finalizado el dictado de clases del año académico 2020 resulta interesante indagar acerca del proceso adaptativo que vivieron los estudiantes y docentes en este año, sus apreciaciones sobre el desarrollo de las clases virtuales, los problemas que enfrentaron y las ventajas y desventajas que percibieron respecto de la modalidad virtual.

Este trabajo examina las percepciones de los estudiantes y docentes sobre la modalidad virtual de enseñanza-aprendizaje implementada en la mayor parte del año 2020. El objetivo 
de la investigación es conocer las valoraciones sobre las modalidades de aprendizaje, reconocer las principales ventajas e inconvenientes percibidas por los estudiantes y docentes respecto de la modalidad virtual de enseñanza y de aprendizaje y su capacidad de adaptación. También se busca profundizar en el conocimiento de las preferencias sobre los formatos de clases y las herramientas didácticas que resultan más atractivas para aprender.

Se trata de un trabajo exploratorio para conocer las opiniones de los estudiantes y su capacidad de adaptación a los cambios en la modalidad de aprendizaje como resultado de la suspensión de las clases presenciales. El trabajo empírico se realiza en la Extensión Áulica San Martín de los Andes de la Facultad Regional del Neuquén de la Universidad Tecnológica Nacional (EASMA-FRN-UTN) donde, en 2020, se dictan dos carreras de tecnicatura: la Tecnicatura Universitaria en Administración (TUA) y la Tecnicatura Universitaria en Energías Sustentables (TUES). El dictado de las carreras comenzó a principios de marzo de 2020 en modalidad presencial y a dos semanas del inicio de clases, éstas quedaron suspendidas en esa modalidad y comienzan a dictarse de manera virtual, con el soporte de un campus virtual de la FRN y clases virtuales sincrónicas mediante diferentes plataformas.

El trabajo se organiza de la siguiente manera. En el siguiente apartado se describe la metodología utilizada. Luego se presentan los resultados del trabajo empírico y, finalmente, se presentan las conclusiones.

\section{METODOLOGÍA}

En este apartado se describe la metodología empleada para alcanzar los objetivos del trabajo exploratorio.

Para recolectar información sobre las percepciones de los estudiantes y docentes se elaboraron sendos cuestionarios que fueron enviados a todos los estudiantes y docentes de las carreras de tecnicatura dictadas en la EASMA-FRN-UTN en 2020, quienes fueron invitados a participar de manera voluntaria. Ambos cuestionarios contaban con preguntas con opciones cerradas de respuesta y otras preguntas de respuesta abierta.

A los estudiantes se les preguntó sobre sus antecedentes con estudios en modalidad virtual, los aspectos de la modalidad virtual con los que estuvieron más y menos a gusto, su preferencia por las clases presenciales y virtuales y las herramientas didácticas que les resultaron más atractivas para aprender. Al final del cuestionario se les hizo una pregunta abierta sobre su percepción general sobre la experiencia vivida en el año en la universidad.

Los docentes fueron indagados sobre sus experiencias previas en la modalidad virtual, los problemas que tuvieron para adaptar sus prácticas docentes a la virtualidad, las herramientas didácticas que utilizaron en sus clases, el desempeño de los estudiantes y sus preferencias por las modalidades presencial y virtual. Como a los estudiantes, al final del cuestionario se les pidió a los docentes que realicen una evaluación general de la experiencia vivida en la universidad durante el año de clases virtuales.

Los cuestionarios se aplicaron entre los meses de octubre y noviembre de 2020. En los siguientes links se encuentran los cuestionarios completos:

- Cuestionario para estudiantes: https://forms.gle/CQsK2DnAtiLPQ32e6.

- Cuestionario para docentes: https://forms.gle/8qHS7ZeGtuhyQMmT7.

Las respuestas fueron procesadas manualmente y los resultados volcados en un software de hoja de cálculo para su análisis. Se procesaron un total de 33 cuestionarios de estudiantes y 11 de docentes. 


\section{RESULTADOS}

En este apartado se presentan y analizan los resultados del estudio empírico realizado en la EASMA-FRN-UTN.

Se recibieron un total de 33 cuestionarios de estudiantes, cantidad que representa el $77 \%$ del total de estudiantes activos de las carreras de TUA y TUES en noviembre de 2020. Asimismo, respondieron el cuestionario respectivo 11 docentes, es decir, todos los que dictaron clases durante 2020 en las carreras mencionadas. Los números y porcentajes que se presentan en esta sección corresponden a los estudiantes y docentes que respondieron los cuestionarios, según corresponda.

\section{ADAPTACIÓN A LA MODALIDAD VIRTUAL}

Cuando se dispone el cese de las clases presenciales, la EASMA-FRN-UTN implementa una modalidad de dictado virtual sincrónica, respetando los horarios de las clases presenciales para las clases a través de plataformas virtuales. Además, se puso a punto el campus virtual de la FRN-UTN, donde estudiantes y docentes accedieron a cada una de las asignaturas de las carreras.

El 73\% de los estudiantes no tenía experiencia previa en realizar estudios en modalidad virtual. Sin embargo, la mayoría se adaptó rápidamente, mientras que el resto (36\%) manifiesta que tuvo ciertas dificultades al comienzo pero logró superarlas (Gráfico 1).

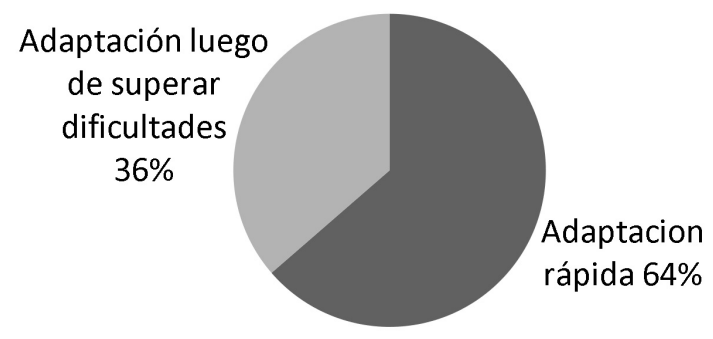

Gráfico 1. Adaptación de los estudiantes a la modalidad virtual (Fuente: Elaboración propia)

En cuanto a los docentes, ninguno tenía experiencia previa en el dictado de clases virtuales. Ante la necesidad de comenzar a hacerlo, el 55\% manifiesta que al comienzo tuvo dificultades pero logró superarlas, mientras que el resto se adaptó rápidamente a la modalidad virtual (Gráfico 2).

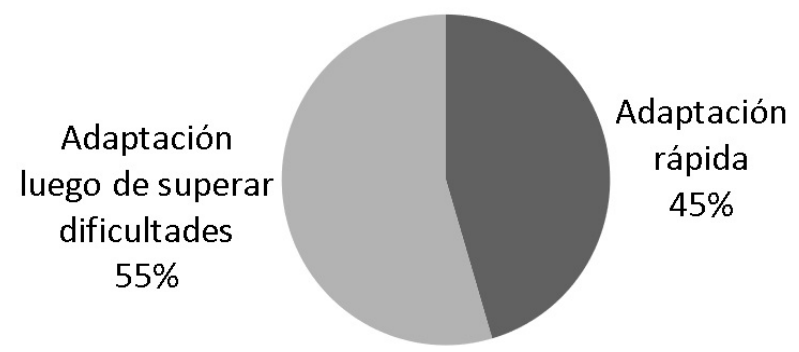

Gráfico 2. Adaptación de los docentes a la modalidad virtual (Fuente: Elaboración propia) 
De las respuestas de los cuestionarios aplicados surge que, tanto docentes como estudiantes, a pesar de la falta de experiencia, se adaptaron a la modalidad virtual, que se extendió hasta la finalización del ciclo lectivo 2020.

\section{PERCEPCIONES SOBRE LAS VENTAJAS DE LA MODALIDAD VIRTUAL}

Los estudiantes fueron indagados acerca sus percepciones sobre las ventajas y desventajas de la modalidad virtual.

Los resultados arrojan que, entre los estudiantes, las principales ventajas percibidas respecto de la modalidad virtual fueron la posibilidad de compatibilizar mejor sus responsabilidades académicas, laborales y familiares y, además, disponer de las clases grabadas para poder volver a verlas y escucharlas cuando lo desearan (Gráfico 3).

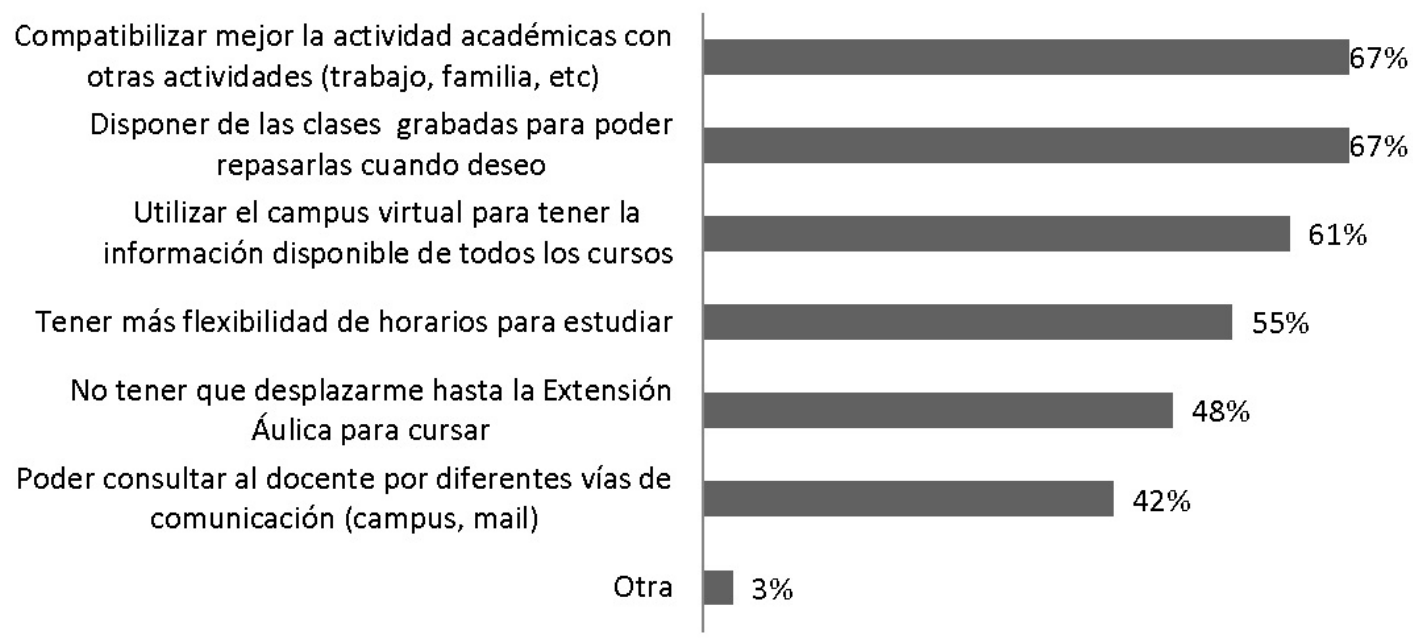

Gráfico 3: Ventajas de la modalidad virtual percibidas por los estudiantes (Fuente: Elaboración propia)

Respecto de la primera, la posibilidad de compatibilizar responsabilidades, cabe destacar que la migración a la modalidad virtual implicó que los estudiantes pudieran estudiar desde sus casas, no haciendo necesario el traslado hasta la facultad y dejando más tiempo disponible para otras actividades.

La implementación de la modalidad virtual se pautó a través de clases sincrónicas respetando los horarios de las clases presenciales según el cronograma establecido al comienzo del ciclo lectivo. Sin embargo, en la práctica se implementó una modalidad donde las clases se dictaban virtualmente en los horarios pautados pero además se grababan y dichas grabaciones quedaban alojadas en el campus virtual y disponibles para todos los estudiantes de la asignatura. Esto implicó que aquellos estudiantes que por diferentes motivos no pudieran conectarse en el horario de la clase, pudieran disponer de ella en el momento que lo desearan, lo cual resulta, junto con la anterior, una de las principales ventajas que los estudiantes encontraron en la implementación de la modalidad virtual. La posibilidad de contar con las grabaciones, en la práctica, flexibilizó el cursado, haciendo más fácil para los estudiantes, compatibilizar compromisos laborales y familiares con los académicos y dándoles la posibilidad de estudiar en los horarios que deseen. En efecto, más de la mitad de los estudiantes 
consideró una ventaja de la modalidad virtual, la posibilidad de tener más flexibilidad de horarios para estudiar.

Otra ventaja observada por los estudiantes, vinculada con la anterior, es la posibilidad de utilizar el campus virtual para tener toda la información disponible de los cursos. El campus, organizado por asignaturas, fue el lugar utilizado por los docentes para compartir los materiales de las clases (incluso las clases grabadas), realizar actividades a través de foros, solicitar tareas, realizar evaluaciones e intercambiar mensajes con los estudiantes.

Las ventajas mencionadas quedan de manifiesto en estas opiniones de los estudiantes recogidas en los cuestionarios:

"La modalidad es interesante y más cómoda porque hay más libertad para organizar los horarios de estudio y, a su vez, los horarios de cada actividad particular de cada estudiante, ya que si algún día no se puede presenciar una clase, ésta esté grabada en el campus para no perderla."

"Al comenzar esta modalidad fue dificil adaptarme, luego supe acomodarme, tener mis tiempos para estudiar y poder enfocarme en los trabajos y proyectos que debía hacer. Fue una gran ventaja tener el material (en el campus) para poder repasar cuando no entendia algún contenido o se me hacia imposible conectarme a clases."

"Fue más cómodo estudiar desde casa, ya que los primeros dias (de clases presenciales) tenía que desplazarme hasta la Extensión Áulica y se me hacía difícil, debido a que no dispongo de vehículo para poder llegar hasta el lugar."

Asimismo, el 94\% de los estudiantes prefiere que las clases queden grabadas para poder consultarlas cuando lo deseen, independiente de la modalidad de dictado de clases (presencial o virtual).

Las ventajas percibidas por los estudiantes coinciden con las encontradas en otras investigaciones (Elacqua y otros, 2020; Goodman y otros, 2017; Deming y otros, 2016). En particular, la evidencia muestra que la educación en modalidad virtual mejora el acceso a la educación al permitir a los estudiantes seguir los cursos a su ritmo, desde el lugar que deseen y en los horarios que mejor se adapten a sus necesidades. Esta mayor flexibilidad fomenta el acceso y la continuidad educativa a quienes, por motivos laborales o familiares, ven dificultado el cumplimiento de sus responsabilidades académicas.

\section{PERCEPCIONES SOBRE LAS DESVENTAJAS DE LA MODALIDAD VIRTUAL}

Los estudiantes también fueron indagados sobre las desventajas que observaron respecto de la modalidad virtual. De los resultados de los cuestionarios aplicados, surge que la principal desventaja observada por los estudiantes fueron los problemas de conectividad (Gráfico 4).

La posibilidad de acceso a internet y de conectividad con una velocidad acorde para poder seguir actividades sincrónicas, se volvieron elementos esenciales para poder estudiar en la EASMA-FRN-UTN así como en el resto de los establecimientos educativos en el año 2020. En Argentina, según datos del ENACOM (Ente Nacional de Comunicaciones, Portal de Datos Abiertos, 2020), el 66\% de los hogares argentinos contaba en 2020 con conexión a internet fija, mientras que en la provincia del Neuquén, ese porcentaje es del 65\%. Asimismo, para toda Argentina, en el grupo etario de 18 a 29 años, el 90\% utiliza internet pero sólo 53,9\% 
utiliza computadora, mientras que el 96\% utiliza teléfonos celulares (Artopoulos, 2020).

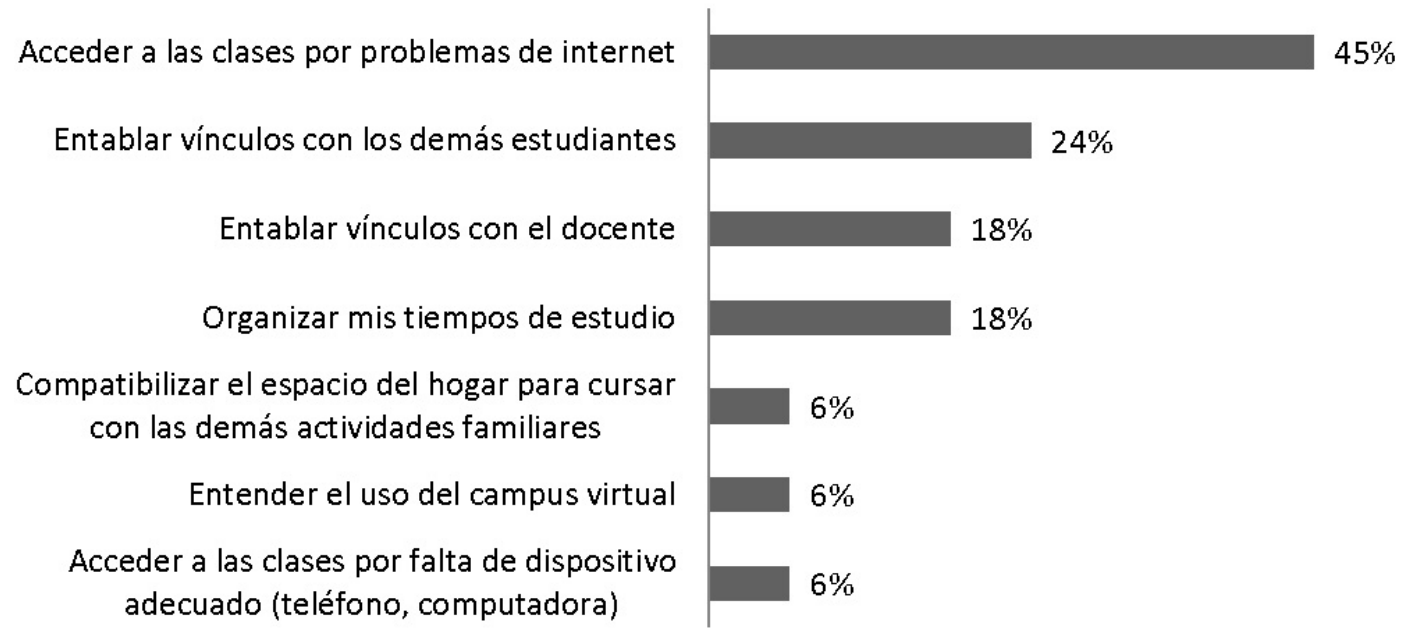

Gráfico 4: Dificultades de la modalidad virtual percibidas por los estudiantes. (Fuente: Elaboración propia)

De acuerdo con los resultados de los cuestionarios aplicados, el 45\% de los estudiantes manifestaron dificultades de acceso a las clases por problemas de internet mientras que sólo el 6\% manifestó dificultades para acceder a las clases por falta de dispositivo adecuado (teléfono, computadora).

Los problemas mencionados supusieron una dificultad inicial que luego fue superada, tal como reflejan las opiniones de los estudiantes en los cuestionarios:

"Al principio me costó mucho adaptarme. Tuve problemas para ingresar a la plataforma y de conectividad por no contar con wifi seguro. Pero, a medida que paso el tiempo y gracias al apoyo de la Universidad, secretaria, compañeros, profesores, pude adaptarme y ponerme al día y me terminó gustando la virtualidad. Me sentí muy acompañada a pesar de estar lejos."

"En un principio me costó adaptarme a la modalidad (virtual), luego de un tiempo y conseguir la computadora para poder estudiar mejoró mucho".

Adicionalmente, casi el 25\% de los estudiantes manifestaron como desventaja vinculada con la modalidad virtual la dificultad para entablar vínculos con los demás estudiantes. Otras desventajas mencionadas fueron las dificultades para organizar los tiempos de estudio y entablar vínculos con los docentes.

Estos resultados coinciden con los encontrados en otros estudios sobre las dificultades para cursar carreras a distancia. Entre los problemas más frecuentes se encuentran las dificultades por factores personales, como la falta de motivación y predisposición al aprendizaje guiado, y también factores vinculados con la experiencia universitaria, como la necesidad de una adecuada organización o método de estudio (Vásquez Martínez, C y otro, 2007). También son relevantes los factores externos al estudiante, como la falta de apoyo familiar, 
conflicto de horarios o no disponer de tiempo suficiente y las dificultades económicas. Además, la educación virtual reduce los elementos de socialización que son importantes para desarrollar habilidades socioemocionales o redes sociales que puedan ser relevantes en el mercado laboral (Elacqua y otros, 2020). Las dificultades para estudiar de manera virtual son incluso causa de abandono de la formación universitaria (Coria, 2020).

\section{PRÁCTICAS DOCENTES Y DESEMPEÑO ESTUDIANTIL EN MODALIDAD VIRTUAL}

Para los docentes, el cambio de modalidad significó un gran desafío, en particular teniendo en cuenta, como se expresó antes, que ninguno tenía experiencia previa en el dictado de clases en modalidad virtual.

Entre las dificultades experimentadas, las principales fueron los problemas para captar la atención de los estudiantes en el entorno virtual y los problemas de conectividad (Gráfico 5).

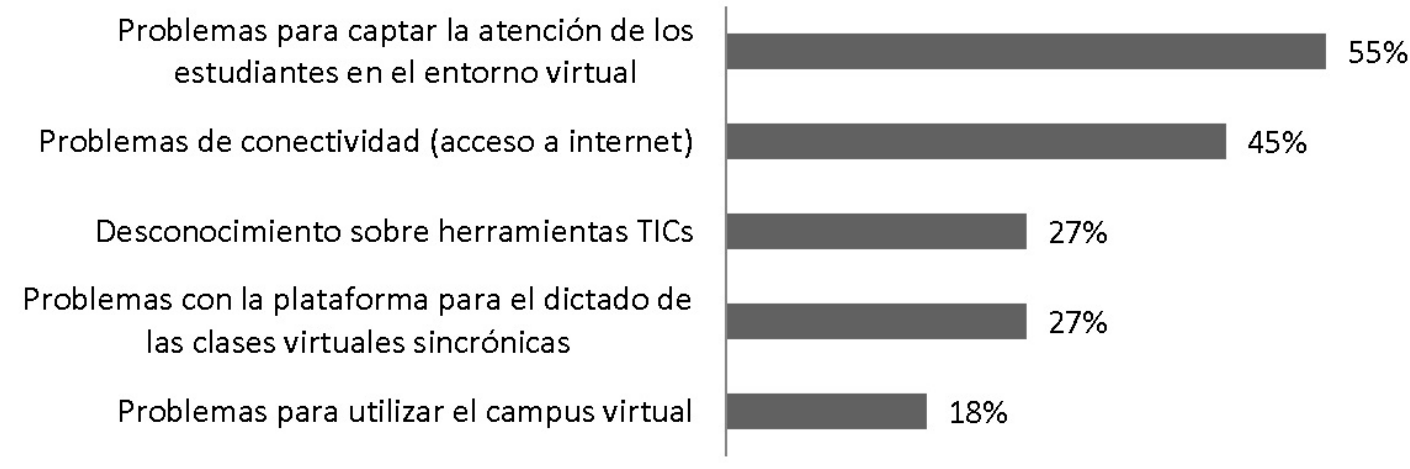

Gráfico 5: Problemas de los docentes para adaptar sus prácticas a la virtualidad . (Fuente: Elaboración propia)

Los problemas para captar la atención se manifiestan en una de las opiniones de los docenes volcadas en los cuestionarios:

"Una experiencia nueva, con el desafio de captar la atención del estudiante. Muchas veces eran "cuadraditos negros" sin una imagen, con la incertidumbre de saber si estaba o no presente. ¿Cómo saber si el estudiante prestaba atención o no? Ante esa situación se apela a la responsabilidad y seriedad de un alumno universitario, y además a la interacción, para "traerlo" desde un audio para confirmar que está."

La dificultad para mantener la atención de los estudiantes es uno de los problemas más observados en los estudios previos (Elacqua y otros, 2020). Las razones fundamentales son que la mayor facilidad de acceso a distracciones a través de internet junto con la falta de control de un docente presencial hace que los estudiantes focalicen menos en sus estudios.

Además, los docentes manifestaron como dificultades los problemas con la plataforma para el dictado de las clases virtuales sincrónicas y el desconocimiento del uso de herramientas TICs. Estos problemas fueron abordados por los docentes adquiriendo nuevas habilidades durante el desarrollo de sus clases, como el uso de plataformas para el dictado de clases, recursos para realizar actividades prácticas y grupales y de evaluación.

Finalmente, tanto estudiantes como docentes plantearon la cuestión de la interacción y 
participación de los estudiantes en clase como una dificultad. En relación con esto, uno de los docentes comenta en el cuestionario:

"Si bien la modalidad virtual tiene muchos aspectos positivos como la posibilidad de acceder con una conexión y trabajar desde cualquier lugar, se "pierde" la dinámica que se genera en un ámbito presencial. La participación de los alumnos y la interacción entre ellos es mucho más notable y enriquecedora con la modalidad presencial que con la virtual."

Asimismo, uno de los estudiantes se manifestó en el mismo sentido:

"(en la virtualidad) se ve limitado el debate o intercambio participativo (con el docente y entre estudiantes), generalmente espontáneo y más dinámico, que suele darse en las clases presenciales."

Como se mencionó antes, al no tener experiencia previa en dictado de clases virtuales, los docentes debieron realizar un importante esfuerzo de adaptación, como menciona un docente en el cuestionario:

"Me llevo un tiempo adaptar el material de estudio y creo que hay mucho que mejorar."

Además de adaptar los materiales, el 82\% de los docentes realizó modificaciones en sus prácticas pedagógicas. En general, los docentes incorporaron nuevas herramientas para el dictado de sus clases, modificaron la metodología de enseñanza, priorizando los contenidos mínimos y plantearon más trabajo interactivo, variando las propuestas y realizaron actividades con menor duración respecto de la presencialidad.

En cuanto a las herramientas didácticas, la más utilizada fue la presentación de los temas con soporte visual (Gráfico 6), y, en menor medida, las imágenes, los videos, los trabajos grupales y la presentación de temas por parte de los estudiantes.

El 36\% de los docentes indicó haber utilizado juegos en sus clases virtuales. El uso de juegos en escenarios no lúdicos (conocido como "gamificación") es una de las estrategias educativas más utilizadas ya que aumenta la motivación de los estudiantes y promueve el aprendizaje (Area y otros, 2014; Mora y otros, 2015). La gamificación no es exclusiva de la modalidad virtual pero sí cobra cada vez más relevancia en estos entornos dado que permite aprovechar los soportes tecnológicos y potencia la captación de la atención de los estudiantes, que es, como se mencionó antes, una de las mayores dificultades de la modalidad virtual de enseñanza. 


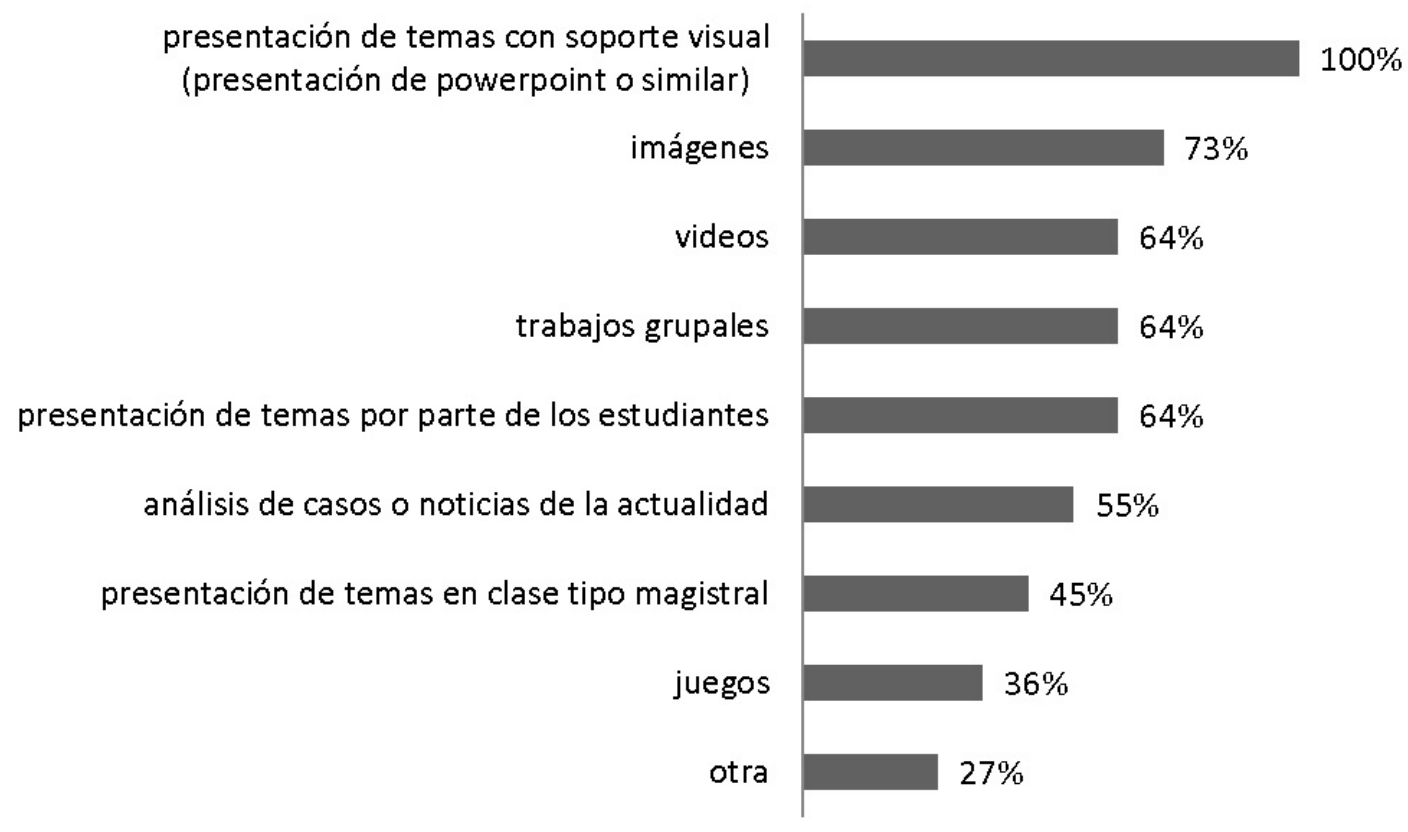

Gráfico 6: Herramientas didácticas utilizadas en las clases en modalidad virtual (Fuente: Elaboración propia)

Respecto al impacto de las prácticas docentes en el desempeño de los estudiantes, más de la mitad de los docentes observó que el desempeño de los estudiantes fue diferente según las herramientas utilizadas en las clases.

En general, los docentes observaron mejor desempeño en las clases donde utilizaron recursos que fomentaron la participación de los estudiantes, como los trabajos en grupo, los juegos, análisis de casos o de películas, debates, foros o presentación de temas por parte de los estudiantes. Es decir, herramientas que aumentan la motivación del estudiante y lo involucran más en el proceso de aprendizaje, otorgándole un rol más activo y participativo. Esta observación coincide con la que realizan los estudiantes: el 97\% de los estudiantes expresa que las clases que más les gustaron son aquellas donde el docente propone un problema o preguntas para que los estudiantes reflexionen.

De las respuestas obtenidas se observa en, en términos generales, los docentes realizan un balance positivo de la virtualidad, tal como surge de los siguientes comentarios realizados en los cuestionarios:

"Mi experiencia fue buena. Implicó un desafío que considero enriquecedor"

"La experiencia fue positiva, la virtualidad permite incursiones en formas de enseñanza y de aprendizaje que de manera presencial las dejamos de lado."

"Fue una experiencia desafiante, pero creo que se lograron cubrir los objetivos de cursado. El balance general es positivo. El grupo de estudiantes puso muy buena disposición y se logró adaptar a la propuesta." 


\section{CONCLUSIONES}

El año 2020 estuvo marcado, al menos en lo que respecta a la educación, por la adaptación a la modalidad virtual de enseñanza y de aprendizaje. La interrupción de las clases presenciales y la adopción de la modalidad virtual significaron importantes esfuerzos tanto para estudiantes como para los docentes.

Este trabajo exploratorio indagó acerca de las percepciones de los estudiantes y docentes con el objetivo de conocer las valoraciones sobre las modalidades de aprendizaje, reconocer las principales ventajas e inconvenientes percibidos por los estudiantes y docentes respecto de la modalidad virtual de enseñanza y de aprendizaje y su capacidad de adaptación. El trabajo empírico se realizó en la Extensión Áulica San Martín de los Andes de la Facultad Regional del Neuquén de la Universidad Tecnológica Nacional (EASMA-FRN-UTN) con los estudiantes y docentes de la Tecnicatura Universitaria en Administración (TUA) y la Tecnicatura en Energías Sustentables (TUES).

Los resultados muestran que, tanto docentes como estudiantes, se adaptaron a la modalidad virtual a pesar de que la mayoría no tenía experiencias previas en esta modalidad de enseñanza y de aprendizaje. En algunos casos, la adaptación tuvo algunas dificultades iniciales pero que lograron ser sorteadas exitosamente.

Las principales ventajas percibidas respecto de la modalidad virtual están relacionadas con la flexibilidad del cursado ya que se percibe a la modalidad virtual como una forma de estudio que permite compatibilizar mejor las responsabilidades académicas, laborales y familiares y, además, estudiar en el horario deseado, volvieron a ver y repasar las clases que quedaban grabadas y alojadas en el campus virtual.

Las prácticas docentes, en su mayoría, debieron ser adaptadas a la nueva modalidad, resultando de dicho proceso de adaptación un mejor desempeño observado por parte de los estudiantes. Los cambios tuvieron que ver, mayormente, con la incorporación de prácticas y recursos que fomenten la atención y participación de los estudiantes, como la presentación de temas con soportes visuales, el uso de imágenes, la presentación de temas por parte de los estudiantes y los trabajos grupales.

Finalmente, cabe destacar que las preferencias por la modalidad virtual son disímiles entre estudiantes y docentes. El 82\% de los docentes está de acuerdo con seguir dictando clases en modalidad virtual, sin embargo, más de la mitad (64\%) prefiere la modalidad presencial. Esta preferencia se explica, en parte, por la percepción de que la modalidad presencial es superior a la virtual en cuanto a las posibilidades de realizar un mejor seguimiento del desempeño de los estudiantes y generar instancias de participación e interacción entre los estudiantes y entre ellos y el docente.

En cambio, entre los estudiantes, el 75\% está de acuerdo en seguir cursando en modalidad virtual y el $46 \%$ la prefiere a la presencial, mientras que el 39\% prefiere la presencial y el resto es indiferente entre ambas modalidades de enseñanza. La preferencia por la virtualidad se explica por la mayor flexibilidad que otorga el cursado en esta modalidad.

Los resultados obtenidos constituyen un primer acercamiento para conocer las preferencias de estudiantes y docentes de la EASMA-FRN-UTN sobre la modalidad virtual que resultó ser novedosa y sin antecedentes entre los estudiantes y docentes de las carreras de grado de la institución.

Por último, los resultados obtenidos refuerzan los encontrados en estudios previos sobre las ventajas e inconvenientes de la modalidad virtual de aprendizaje y permiten conocer, a partir de las percepciones, las oportunidades y desafíos que presenta esta modalidad de enseñanza que se vislumbra con un fuerte potencial para mejorar el acceso a la educación superior. 


\section{REFERENCIAS}

Area, M; González González, C; Mora, C (2014) "Beyond Textbooks: Educational Digitals Texts and Gamification of Learning Materials" Capítulo del libro "Digital textbooks: What's new?" de Rodríguez, J; Bruillard, E y Horsley, M (editores). Editorial de la Universidad de Santiago de Compostela. Disponible en https://www.researchgate.net/publication/270882725_Beyond_Textbooks_Educational_Digitals_Texts_and_Gamification_of_Learning_Materials

Artopoulos, A (2020) “Acceso a internet. ¿Cuántos estudiantes tienen acceso a internet en su hogar en Argentina?”. Informe del Observatorio Argentinos por la Educación. Disponible en https:// cms.argentinosporlaeducacion.org/media/reports/ArgxEdu_Conectividad_Coronavirus_.pdf

Coria, M (2020) "Factores que influyen en la decisión de ingreso a la Universidad". Revista Tecnología Y Ciencia, (39), 178-194. ISSN 1666-6933. Disponible en https://doi.org/10.33414/ rtyc.39.178-194.2020

Deming, D; Lovenheim, M y Patterson, R (2016) “The competitive effects of online education" National Bureau of Economic Research (NBER) WP 22749. Disponible en http://www.nber.org/ papers/w22749

Elacqua, G, Navarro-Palau, P, Prada, M y Soares, S (2020) "Educación a distancia, semipresencial o presencial. ¿Qué dice la evidencia?” Hablemos de Política Educativa $\mathrm{N}^{\circ} 5$. Banco Interamericano de Desarrollo (BID). Disponible en https://publications.iadb.org/publications/spanish/ document/Hablemos-de-politica-educativa-en-America-Latina-y-el-Caribe-5-Educacion-a-distancia-semipresencial-o-presencial-Que-dice-la-evidencia.pdf

Ente Nacional de Comunicaciones (2020) Portal de Datos Abiertos. Disponible en http://datosabiertos.enacom.gob.ar/dashboards/20000/acceso-a-internet/

Goodman, J; Melkers, J y Pallais, A (2017) "Can online delivery increase access to education?” National Bureau of Economic Research (NBER) WP 22754. Disponible en http://www.nber.org/ papers/w22754

Ministerio de Educación de la Nación (2017) “Estadísticas Universitarias”. Secretaría de Políticas Universitarias. Disponible en http://estadisticasuniversitarias.me.gov.ar/\#/home

Mora, A; Riera, D; González González, C (2015) "A Literature Review of Gamification Design Frameworks" 7th International Conference on Games and Virtual Worlds for Serious Applications (VS-Games). Skövde, Suecia. Disponible en https://www.researchgate.net/publication/279059823_A_Literature_Review_of_Gamification_Design_Frameworks

Rieble-Aubourg, S y Viteri, A (2020) “COVID-19: ¿Estamos Preparados para el Aprendizaje en Línea?”, Nota CIMA, No. 20, Washington, D.C., Banco Interamericano de Desarrollo (BID). Disponible en https://publications.iadb.org/publications/spanish/document/Nota-CIMA--20-COVID-19-Estamos-preparados-para-el-aprendizaje-en-linea.pdf

Vásquez Martínez, Cy Rodríguez Pérez, M.C. (2007) “La deserción estudiantil en educación superior a distancia: perspectiva teórica y factores de incidencia”. Revista Latinoamericana de Estudios Educativos, 37 (3-4), pp. 107-122. Disponible en https://www.redalyc.org/pdf/270/27011410005. pdf 